目

\title{
Co-simulation of the virtual vehicle in virtual traffic considering tactical driver decisions
}

\author{
Jakob Kaths $^{1 *}$, Benedikt Schott ${ }^{1}$ and Frederic Chucholowski ${ }^{1}$ \\ ${ }^{1}$ Vector Informatik GmbH, Niederlassung München, Germany \\ jakob.kaths@vector.com, benedikt.schottevector.com, \\ frederic.chucholowski@vector.com
}

\begin{abstract}
Recent developments such as increasing automation and connectivity of vehicles as well as new regulations for real driving emissions lead to a stronger consideration of traffic and traffic control in automotive development. The increasing complexity of vehicular systems requires a highly virtualized development process. Therefore, a cosimulation solution of DYNA4's virtual vehicle with SUMO's microscopic traffic is presented here. Despite increasing automation, virtual test drives often still require a virtual test driver. Thus, the co-simulation solution is extended by combining the driver models of both tools. The operational decision making level of DYNA4 is extended by SUMO's tactical driver decisions, aiming at virtual test drives in complex surrounding traffic with realistic reaction on traffic and traffic control and reduced parametrization effort. By comparing two variants it is shown that a higher reference speed and more aggressive lane change parameters lead to an increase of usage of the left lane and an increase in achieved speeds.
\end{abstract}

\section{Introduction and motivation}

Increasing complexity of vehicular systems leads to an ever increasing need for testing during the development process. At the same time, development time and costs should be limited, making the intensive use of simulation paramount. With progressing automation and connectivity of vehicles, the consideration of surrounding traffic and traffic control in the test and development of automotive systems is gaining higher importance. Well-proven control systems for driving dynamics, like electronic stability control, are tested within completely static environments according to standard maneuvers such as the sine-with-dwell defined in ECE-R 13-H and its respective EU regulation (Economic Commission for Europe of the United Nations, 2018). For driver assistance systems, prescribed maneuvers such as the NCAP catalogue for automatic emergency braking exist

* corresponding author

M. Weber, L. Bieker-Walz, R. Hilbrich and M. Behrisch (eds.), SUMO2019 (EPiC Series in Computing, vol. $62)$, pp. $21-28$ 
(EuroNCAP, 2019). These include only few surrounding road users with relatively simple, deterministic maneuvers. For automated and connected driving, however, no such regulations or test catalogues exist yet in the public domain. Furthermore, the complexity of test scenarios is likely to be much higher and comprises a larger parameter space as the complexity of driving functions and their range of applicability is largely increased as well. For certain use-cases, especially in the earlier development phases, free driving in stochastically generated scenarios can address the lack of an exhaustive catalog of standard scenarios. Therefore, the vehicle and environment simulation DYNA4 is extended with an interface for co-simulation with the microscopic traffic simulator SUMO (Krajzewicz, Erdmann, Behrisch, \& Bieker, 2012). This coupling is described in the first part of this paper. In the second part, the co-simulation is extended towards the virtual test driver. The operational decision making level of DYNA4 is extended by SUMO's tactical driver decisions, aiming at virtual test drives in complex surrounding traffic with realistic reaction on surrounding road users and traffic control at a reduced parametrization effort. Bearing in mind the detailed environment simulation of DYNA4, a non-exhaustive list of possible evaluation use-cases could be given as follows:

- performance of (partially) automated driving functions in complex surrounding traffic,

- performance of sensor processing functions such as object recognition,

- effect of traffic and traffic control on efficiency-related vehicle functions,

- and effects of traffic control or infrastructure measurements on automated vehicles or human drivers.

Especially the second and third use-case can benefit strongly from the introduction of a virtual test driver that includes tactical and operational actions.

\section{Simulation of the virtual vehicle in virtual traffic}

Different development tasks in automotive and traffic engineering led to well-established simulation tools in these engineering domains. Vehicle and traffic simulators of course differ a lot, despite the fact that the movement of vehicles is of interest in both disciplines. In this chapter, vehicle and traffic simulation tools are briefly compared on a general level. Subsequently, the co-simulation solution of DYNA4 and SUMO is explained - both tools are chosen as representatives for their respective domain. Although a number of publications from the authors of this paper and others already exist (Kaths \& Krause, 2016; Semrau, Erdmann, \& Friedrich, 2018; Wiegel, 2017), none of these solutions seems to be publicly available. Furthermore, an extension of the co-simulation towards driver models, as presented in this paper, is usually not included. Parts of the following explanations are based on a German publication from the authors of this paper (Kaths, Schott, \& Chucholowski, 2018).

\subsection{Comparison of vehicle and traffic simulators}

Chassis movements, tire forces and turning speeds are examples for necessary signals that are of interest in automotive engineering for the development of control algorithms. This development can be done completely virtually (Model-in-the-Loop) or including some of the components as actual hardware (Hardware-in-the-Loop). Usually, a single vehicle with multi-body systems for the axles, with tire models, powertrain models and sensors is simulated to deliver signals in the desired accuracy. Especially in the case of Hardware-in-the-Loop setups, it is necessary to calculate these models faster than real-time to supply controllers with updated signals timely. In traffic engineering, on the other hand, typical questions to be addressed by simulation are the influence of traffic control 
on the flow of traffic as a whole. Relevant measurements are, for example, travel times or the number of stops. In microscopic traffic simulations, a human-like car-following behavior is simulated together with models for lane-changing. Because the model of a single vehicle is less detailed than in the case of vehicle simulation, it is possible to simulate hundreds of vehicles faster than real-time. Similar to the X-in-the-Loop approaches in automotive engineering, it is common practice to test traffic control algorithms with traffic simulations. A major difference between vehicle and traffic simulations lies in the evaluation of results. Vehicle simulation relies on exact and deterministic reproducibility to efficiently evaluate the effects on one vehicle under test (VuT) in a large parameter space. Microscopic traffic simulation, on the other hand, incorporates stochastic behavior to account for realworld variations. Each scenario, therefore, is simulated multiple times and subsequently compared with statistical methods (FGSV, 2006). The selection of random seeds still allows for an exact reproducibility of traffic simulations. The difference in the level of detail of models and the requirements of signal update rates of the systems under test lead to a large difference in the step size of vehicle $(\sim 1000 \mathrm{~Hz})$ and traffic simulation $(\sim 1 \mathrm{~Hz}$ to $10 \mathrm{~Hz})$. The different levels of detail also lead to different requirements regarding the road model. As an example, the cross fall of a road only has limited effects on the traffic flow, but is relevant for driving dynamics.

\subsection{Coupling DYNA4 and SUMO}

The road network description builds the static basis for the co-simulation of DYNA4 and SUMO. Without conversion, DYNA4 uses the standard OpenDRIVE (ASAM e.V., 2019), while SUMO allows for straight-forward conversion of OpenDRIVE files into the SUMO network description format via NETCONVERT. In contrast to the prototype solution presented by Kaths \& Krause (2016), even highly complex road networks are easily exchanged between both tools and mutually used. Ideally, OpenDRIVE is used as the data source, since a richer information basis is delivered. If necessary, manual changes and additions can easily be made to the resulting SUMO network with NETEDIT. As such, traffic signal control information can be provided in NETEDIT that will be synchronized with DYNA4 in the co-simulation setup.
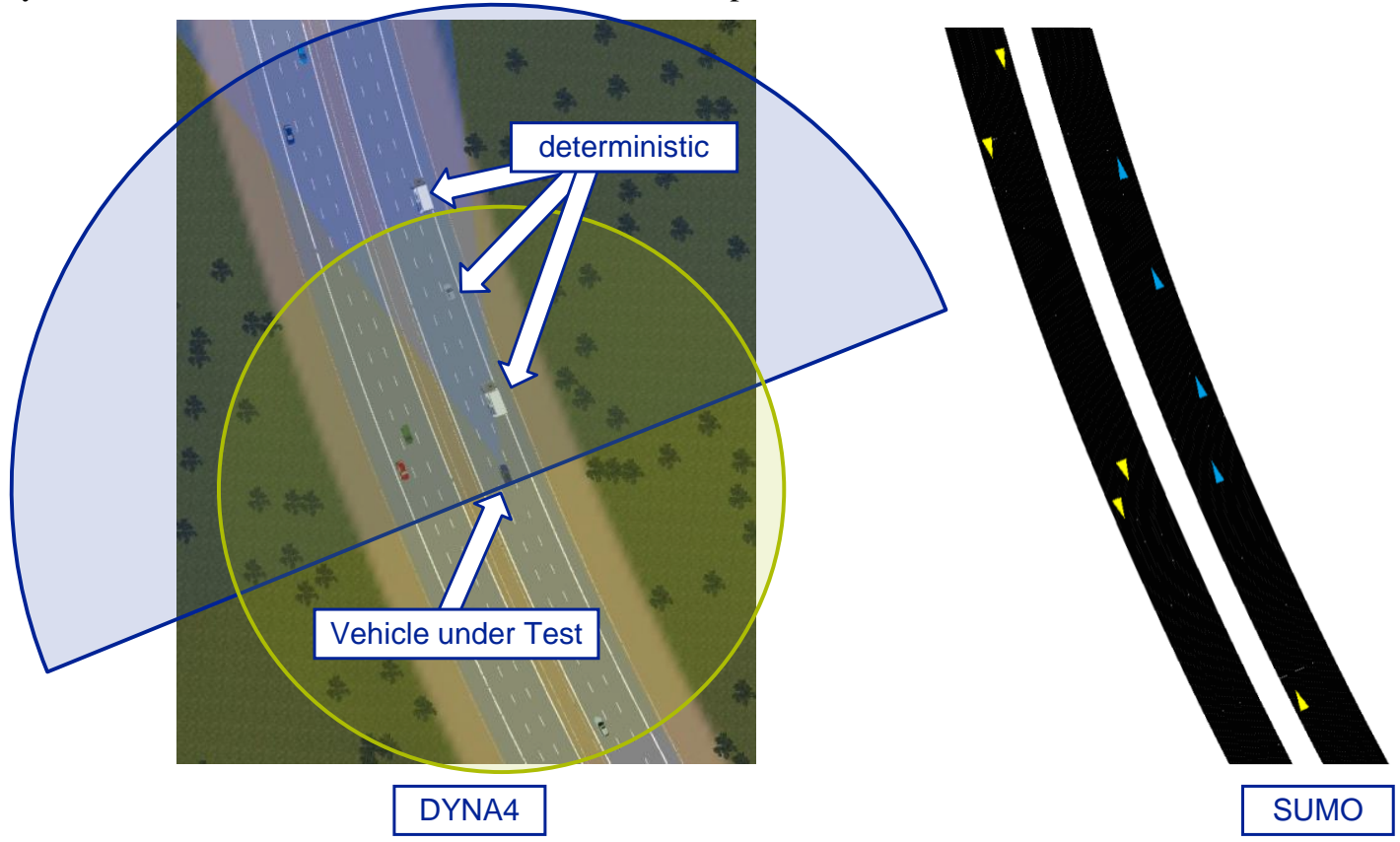

Figure 1: Co-simulation of a freeway scenario in DYNA4 (left) and SUMO (right) 
To synchronize traffic signal information between SUMO, DYNA4 and OpenDRIVE, generic parameters are added to the junction as a key and value pair. The key stores the signal name that is valid for DYNA4 and OpenDRIVE, while the value stores the corresponding SUMO "link tls index".

Building on this mutual static information basis, a dynamic exchange of information is necessary for establishing the co-simulation between both tools. DYNA4's models are Simulink-based, which allows for an efficient integration of $\mathrm{C}++$ code via S-functions. Therefore, the performant $\mathrm{TraCI} \mathrm{C}++$ API of SUMO is used to retrieve data from and send data to SUMO. In short, this data exchange will:

- initially place and subsequently move the VuT and possible other road users with deterministic maneuvers

- collect information of road users (cars, trucks, busses, pedestrians, bicycles) surrounding the VuT including their type, speed, position etc.

To perform the latter in the most efficient way, a context subscription is used to continuously retrieve the entire set of data from all surrounding road users in a circle (see yellow circle in Figure 1) without having to issue several time consuming TraCI communication commands. Because often the road users in driving direction are of higher relevance than the ones behind the VuT, a second subscription is used of which only the road users in the front semi-circle are considered (see blue semi-circle in Figure 1). Additionally to the VuT, it is possible to plan the maneuvers of deterministically controlled road users in DYNA4, e.g. in order to provoke a critical cut-in maneuver, see highlighted vehicles in Figure 1.

The larger step size of SUMO is one of the challenges that need to be addressed in the cosimulation setup. This is done by means of a slowest-first approach: a SUMO step is calculated in advance and the values are interpolated subsequently for the trailing calculations of DYNA4. The direct usage of these interpolated values, however, would not lead to satisfactory movements of the road users especially with regard to chassis movements. Therefore, the interface to the DYNA4 traffic component, which typically serves for the generation of deterministic road users, was opened for external control signals. As such, SUMO delivers initial guesses for positions and speeds and DYNA4's traffic component smoothens the signals and enhances them with yaw, roll and pitch. Furthermore, a placement on the OpenDRIVE road is performed, which leads to plausible behavior even when a cross fall is present. Another benefit of this procedure is the full availability of features for road users in DYNA4, including their visibility for Simulink-based sensors. The process is depicted in a simplified way in Figure 2.

To generate plausible vehicle movements and to achieve the desired display of road users in the 3D animation, a number of additional parameters has to be provided for each vehicle that go beyond the available parameters in SUMO. While vehicle dimensions (height, width, length) and vehicle class (passenger car, pedestrian, etc.) can be used directly from SUMO, each road user has to carry additional information that is relevant for the correct visualization and movement modelling in DYNA4. This information is stored with predefined keywords in SUMO's type IDs and can, therefore, be accessed for each vehicle. This additional information includes:

- $\quad$ which geometry to visualize,

- tire radius,

- wheel base and

- distance between front axle and bumper.

It is planned to transfer these parameters into generic parameters in SUMO to make them accessible in an easier way through $\mathrm{TraCI}$ and to increase transparency for the user. 


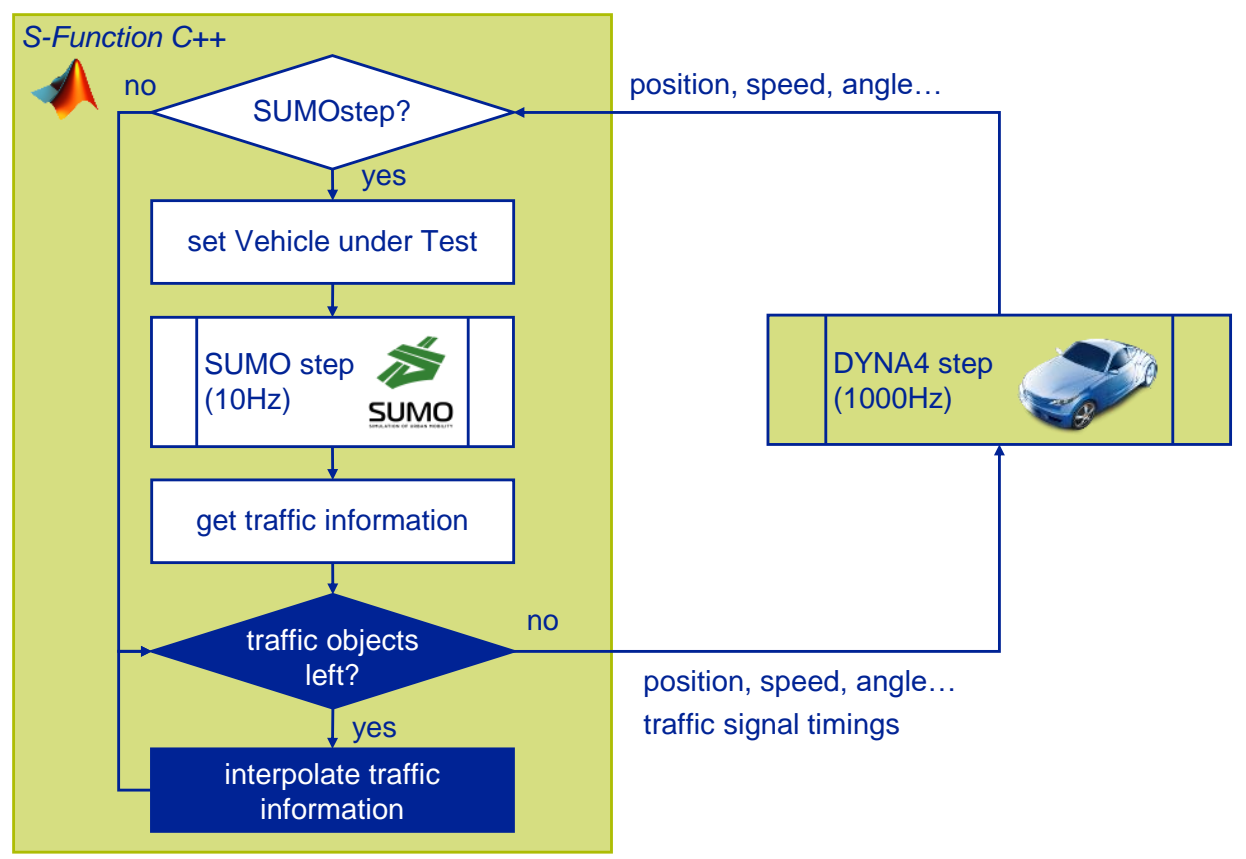

Figure 2: Flowchart depicting the co-simulation procedure of DYNA4 and SUMO

Generally, the number of vehicles considered in the Simulink model and in the 3D animation is not limited. For performance reasons, a limitation to 40 surrounding road users is implemented here. It is important to notice that each of the 40 road users can switch vehicle classes and animation geometries over time. Therefore, the performance is mainly determined by the 3D animation, which has to provide each object geometry for each of the 40 possible objects. In case the SUMO subscription delivers more than 40 surrounding road users, only the 40 closest road users are considered and displayed. In this constellation, even complex scenarios can be simulated in faster than real-time on standard desktop computers, making it possible to use real-time capable execution platforms such as Vector CANoe, if necessary. The solution presented here is, therefore, much more performant and efficient than the prototype presented earlier by Kaths \& Krause (2016).

\section{Extending the co-simulation towards the virtual driver}

As stated in chapter 1, despite the fact that the proposed co-simulation setup aims at the development of automated driving functions, often a virtual test driver is necessary that is able to cope with complex traffic situations efficiently. For example, the perception level with object detection and sensor fusion algorithms could be evaluated independently from the actual driving function. DYNA4 offers models for virtual test drivers with focus on the operational level. The task of stabilizing the car (see Donges (1982)) is performed by these models longitudinally and laterally. The decisions are transformed into a realistic human-like actuation of pedals and steering wheel. Tasks that can be performed in this way are route and lane following, following other traffic participants, reacting to speed limits and so on. However, the more tactical or even strategical levels such as the navigation level and the trajectory decision making have to be parametrized partially manually. For these cases, the co-simulation solution is extended towards the virtual driver by taking such higher level decisions 
from SUMO. To this end, the relevant information from the VuT is received and output and can be used subsequently in the Simulink model of DYNA4. The example that is highlighted in this paper is the tactical change of lanes, but the same principle can be used for other variables as well. Here, the command "getLaneChangeState" is performed at each simulation step for the VuT and the received information triggers lane changes whenever desired and possible. To avoid oscillations and problems that could be caused by short mismatches between lane information from SUMO and DYNA4 the signals stemming from SUMO are debounced. As such, lane change requests from SUMO are only used if active for certain period then held constant to securely trigger the lane change in DYNA4. Until the lane change is performed completely in DYNA4, no further lane change requests are considered.

\section{Simulation study}

Using the example of an automated drive on a freeway, this simulation study intends to demonstrate the co-simulation of DYNA4 and SUMO itself, but also the usage of tactical driver decisions from SUMO for the VuT in DYNA4. For this example, a $5 \mathrm{~km}$ long stretch of the German freeway A9 north of Munich is used. This OpenDRIVE file has been created by 3DMappingSolutions based on precise surveying. For longitudinal control, the exemplary ACC module of DYNA4 is used. The lateral control on the operational level is performed by DYNA4's virtual driver model, which is set to follow a given lane. This lane information, however, is not static, but retrieved from TraCI and smoothened as described above. To evaluate the effect of the introduction of SUMO's lane change behavior into DYNA4, the variations "slow" and "fast" for the settings of the VuT are given in Table 1.

\begin{tabular}{lrrrr}
\hline variation & $\begin{array}{r}\text { ACC ref speed } \\
\mathrm{m} / \mathrm{s}\end{array}$ & $\begin{array}{r}\text { maxSpeed } \\
\mathrm{m} / \mathrm{s}\end{array}$ & $\begin{array}{r}\text { lcStrategic } \\
\text { lcKeepRight }\end{array}$ \\
\hline slow & 25 & 25 & 0.1 & - \\
fast & 50 & 50 & 100 & 100 \\
& & & & 1
\end{tabular}

Table 1: Variations of settings for the vehicle under test

The variation "slow" incorporates a slower reference speed for the ACC system set in DYNA4 and less aggressive lane change model parameters for strategic lane changes (SUMO parameter: lcStrategic) and the eagerness to keep right (SUMO parameter: lcKeepRight). The maximum speed for the corresponding SUMO vehicle is set to the same speed as the ACC reference speed. For the "fast" variation, the reference speed is doubled to $50 \mathrm{~m} / \mathrm{s}$ and lane change parameters are set more aggressively. For convenience, these values are set during the simulation with a second TraCI client via a short Python script. Both variations are evaluated in surrounding SUMO traffic with a flow rate of $5400 \mathrm{veh} / \mathrm{hr}$ on three lanes. The times of presence on the right, center and left lane of the VuT as well as its mean speed are recorded with the DYNA4 trace mechanism and postprocessed for evaluation of the co-simulated lane change behavior. For each variation, 10 simulation runs are performed and the mean values are used.

Figure 3 shows the lane usage of the vehicle under test. As expected, the distribution of the lane usage shifts heavily from the right to the left lane when choosing a higher maximum speed and allowing for more lane changes. In the "slow" variant, the right lane is used the majority of the trip with about $62 \%$ of the time. This value decreases to about $7 \%$ in the "fast" variant. In a reciprocal way, the usage of the left lane increases from about $10 \%$ to over $70 \%$. 
As a result, the achieved mean speeds increase for the "fast" variant from $24.5 \mathrm{~m} / \mathrm{s}$ to $31.5 \mathrm{~m} / \mathrm{s}$, see Figure 4. The reason for the relatively big difference between the reference speed and the achieved speed in case of the "fast" variant is the impact of the surrounding and the chosen vehicle configuration with relatively low acceleration capabilities.

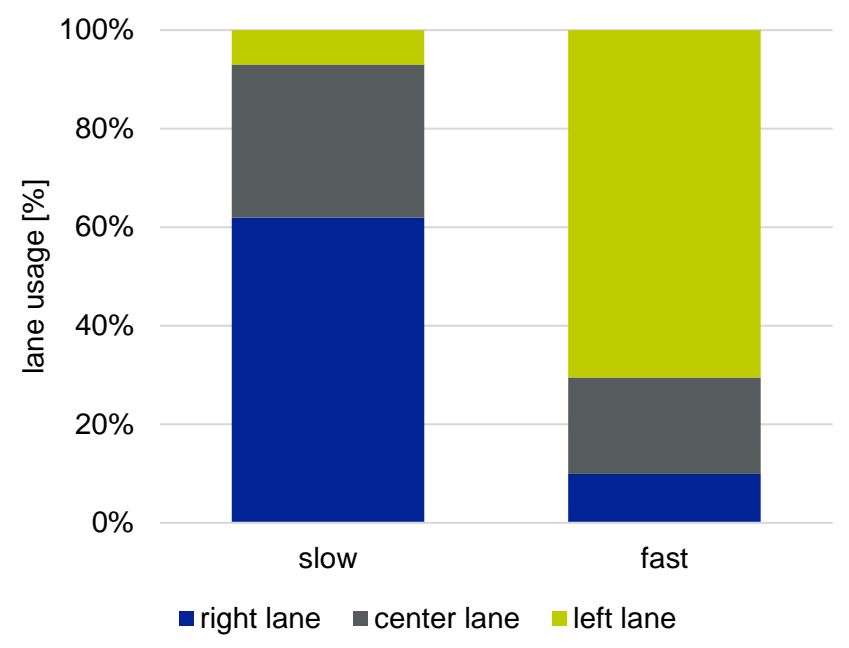

Figure 3: Lane usage of the vehicle under test

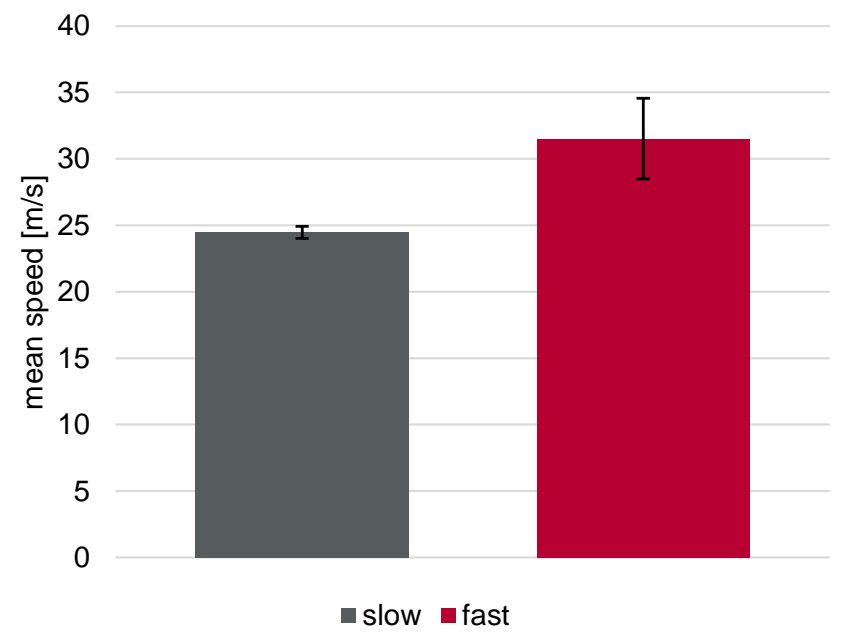

Figure 4: Mean speed of the vehicle under test

The results indicate, that it is feasible to enhance the operational driver model of DYNA4 by introducing tactical decisions of SUMO's lane change models. By influencing the SUMO lane change parameters, a parametrization of the driver of the VuT is possible. 


\section{Conclusions and outlook}

In this paper, a number of interesting use cases for the combined usage of vehicle and traffic simulation are given. A co-simulation solution based on DYNA4 and SUMO is presented that addresses these use-cases. The setup allows for an efficient generation of complex scenarios while maintaining real-time capabilities for the test and development of demanding vehicle functions. The co-simulation is extended beyond the exchange of vehicular information by including the exchange of driver decision information. In this way, the parametrizable lane change model of SUMO can be used to enhance the operational driver model of DYNA4.

The demonstrated functionalities and procedures could be adapted in future work to cover urban scenarios. Besides lane changes due to turning maneuvers the speed adaptations for yielding at signalized or unsignalized intersections would be of high interest.

The work carried out for this publication was performed with SUMO 1.1.0 and DYNA4 3.0. The SUMO integration package is readily available for DYNA4 and support for future SUMO usually requires only minor adaptations due to changes in the TraCI API. Support for SUMO 1.2.0 will be given with DYNA4 3.1. Simulations in the current configuration are real-time capable. However, great performance improvements could be expected by reducing or completely avoiding the need of socket communication with TraCI. Therefore, the usage of the libsumo functionality (DLR, 2019) is intended for future implementations.

\section{References}

ASAM e.V. (2019). ASAM OpenDRIVE. Retrieved March 5, 2019, from https://www.asam.net/standards/detail/opendrive/

DLR. (2019). Libsumo. Retrieved from https://sumo.dlr.de/wiki/Libsumo

Donges, E. (1982). Aspekte der Aktiven Sicherheit bei der Führung von Personenkraftwagen. Automobil-Industrie, 2, 183-190.

Economic Commission for Europe of the United Nations. Regulation No 13-H of the Economic Commission for Europe of the United Nations (UN/ECE) — Uniform provisions concerning the approval of passenger cars with regard to braking (2018). European Union.

EuroNCAP. Test Protocol - AEB VRU systems v 2.0.4 (2019).

FGSV. (2006). Hinweise zur mikroskopischen Verkehrsflusssimulation - Grundlagen und Anwendung.

Kaths, J., \& Krause, S. (2016). Integrated simulation of microscopic traffic flow and vehicle dynamics. In IPG Apply \& Innovate. Karlsruhe.

Kaths, J., Schott, B., \& Chucholowski, F. (2018). Integration des virtuellen Fahrzeugs in die mikroskopische Verkehrsflusssimulation zur virtuellen Erprobung kooperativer Systeme. In SIMVEC. Baden-Baden.

Krajzewicz, D., Erdmann, J., Behrisch, M., \& Bieker, L. (2012). Recent development and applications of SUMO--simulation of urban mobility. International Journal On Advances in Systems and Measurements, 5(3\&4).

Semrau, M., Erdmann, J., \& Friedrich, B. (2018). Multi-Level-Validation of Chinese traffic in the ChAoS framework. In SUMO 2018 - Simulating Autonomous and Intermodal Transport Systems. Berlin.

Wiegel, A. (2017). PTV Group und IPG Automotive bieten neue Schnittstelle für den virtuellen Fahrversuch. Retrieved August 17, 2018, from http://compass.ptvgroup.com/2017/03/ptv-undipg-automotive-bieten-neue-schnittstelle-fuer-den-virtuellen-fahrversuch/ 